\title{
Recuperação funcional em cães com doença do disco intervertebral toracolombar sem percepção à dor profunda: 37 casos $(2002-2010)^{1}$
}

\author{
Rosmarini P. Santos ${ }^{2}$, Alexandre Mazzanti ${ }^{*}$, Diego V. Beckmann², Larissa \\ Berté$^{2}$, Angel Ripplinger ${ }^{4}$, Dakir Polidoro Neto $^{4}$ e Raquel Baumhardt ${ }^{4}$
}

\begin{abstract}
Santos R.P., Mazzanti A., Beckmann D.V., Berté L., Ripplinger A., Neto D.P. \& Baumhardt R. 2011. [Functional recovery in dogs with thoracolumbar intervertebral disk disease without deep pain perception: 37 cases (2002-2010).] Recuperação funcional em cães com doença do disco intervertebral toracolombar sem percepção à dor profunda: 37 casos (2002-2010). Pesquisa Veterinária Brasileira 31(4):345-349. Departamento de Clínica de Pequenos Animais, Centro de Ciências Rurais, Universidade Federal de Santa Maria, Av. Roraima 1000, Camobi, Santa Maria, RS 97105-900, Brazil. E-mail: alexamazza@yahoo.com.br

The aim of this study was to evaluate functional recovery in 37 cases with diagnostic of thoracolumbar intervertebral disk disease in dogs without deep pain perception (more than 48 hours) and did not underwent surgical treatment from 2002 to 2010 . The following data were obtained: Breed, age, sex, neuroanatomic localization, loss of deep pain perception, duration of clinical signs, functional recovery, deep pain recovery, recurrence and euthanasia or death. A satisfactory functional recovery was observed in 11 dogs $(55 \%)$, mostly between 30-60 days after the beginning of the clinics signal (six dogs). Two of 11 dogs with satisfactory functional recovery did not recovered deep pain perception. The results showed that dogs with presumptive diagnoses of thoracolumbar intervertebral disk disease with more than 48 hours and that did not underwent surgical treatment are capable of a functional satisfactory recovery and should be waited 30 days after clinical signs begin to establish a prognosis on the recovery of voluntaries movements.
\end{abstract}

INDEX TERMS: Diseases of dogs, intervertebral disk disease, paraplegia, functional recovery, neurology.

RESUMO.- O objetivo deste trabalho foi avaliar a recuperação funcional de 37 cães com diagnóstico de doença do disco intervertebral (DDIV) toracolombar, sem percepção da dor profunda superior a 48 horas e não submetidos ao tratamento cirúrgico. Os dados identificados foram: raça, idade, sexo, localização da lesão, perda da percepção da dor profunda, duração dos sinais clínicos, recuperação funcional, retorno da percepção da dor profunda, recidivas, eutanásias ou morte. Foi observada recuperação funcio-

\footnotetext{
${ }^{1}$ Recebido em 14 de agosto de 2010.

Aceito para publicação em 26 de outubro de 2010.

2 Programa de Pós-Graduação em Medicina Veterinária, Área de concentração em Cirurgia, Centro de Ciências Rurais (CCR), Universidade Federal de Santa Maria (UFSM), Av. Roraima 1000, Camobi, Santa Maria, RS 97105-900, Brasil.

3 Departamento de Clínica de Pequenos Animais, CCR-UFSM. "Autor para correspondência: alexamazza@yahoo.com.br

${ }^{4}$ Curso de Medicina Veterinária, CCR-UFSM, Santa Maria, RS.
}

nal em 11 cães (55\%), sendo seis deles entre 30 e 60 dias após o início dos sinais clínicos. Dos onze cães que tiveram recuperação funcional satisfatória, dois (18\%) não tiveram retorno da percepção à dor profunda. Pode-se concluir que cães com diagnóstico de DDIV sem percepção à dor profunda superior a 48 horas e não submetidos ao tratamento cirúrgico podem apresentar recuperação funcional satisfatória e são necessários, no mínimo, 30 dias do início dos sinais clínicos para estabelecer um prognóstico quanto ao retorno dos movimentos voluntários.

TERMOS DE INDEXAÇÃO: Doenças de cães, doença do disco intervertebral, paraplegia, recuperação funcional, neurologia.

\section{INTRODUÇÃO}

As discopatias constituem uma das causas mais comuns de disfunção neurológica em cães, ocasionadas pela protusão (Hansen II) ou extrusão (Hansen I) de material do disco intervertebral para o interior do canal vertebral com subsequente 
compressão da medula espinhal (Wheeler \& Sharp 1999, Lecouteur \& Grandy 2004, Seim 2005, Dewey 2006).

As extrusões afetam predominantemente cães de raças condrodistróficas, com idade entre três e seis anos, e ocorrem mais frequentemente na região cervical, toracolombar (T11-L3) e lombar (Wheeler \& Sharp 1999, Lecouteur \& Grandy 2004, Taylor 2006). Ocorrem de forma aguda e a gravidade da lesão depende de vários fatores, como a velocidade com que a força compressiva é aplicada, o volume da massa compressiva e a duração da compressão (Toombs \& Bauer 1998, Lecouteur \& Grandy 2004). Os sinais clínicos podem ser agudos ou crônicos, variando desde hiperestesia espinhal até paraplegia e perda da percepção da dor profunda, este último, considerado indicador confiável de prognóstico em lesões compressivas da medula espinhal (Toombs \& Bauer 1998, Lecouteur \& Grandy 2004, Taylor 2006).

O diagnóstico da doença do disco intervertebral (DDIV) é estabelecido através de sinais clínicos, história clínica, exame físico e neurológico (Tooms \& Bauer 1998) e da realização de exames radiográficos simples (Wheeler \& Sharp 1999). Os diagnósticos diferenciais da DDIV incluem: discoespondilite, mielites, traumatismo vertebral, neoplasmas (vertebrais, medulares ou do canal vertebral), distúrbios congênitos (vertebrais ou medulares) e mielopatia isquêmica (Wheeler \& Sharp 1999).

O tratamento clínico para cães com DDIV é recomendado aos pacientes somente com hiperestesia ou com deficiências neurológicas discretas (Lecouteur \& Grand 2004) e consiste no confinamento em gaiola durante três a quatro semanas, com ou sem o uso de anti-inflamatórios (Dewey 2006). O tratamento cirúrgico é indicado para pacientes com recidiva ou progressão dos sinais, paraparesia não ambulatória, paraplegia com preservação da dor profunda ou sua ausência com menos de 48h (Sharp \& Wheeler 2005).

Vários artigos têm demonstrado a taxa de recuperação funcional de cães com DDIV sem percepção da dor profunda com duração igual ou superior a 48 horas e que foram submetidos ao tratamento cirúrgico (Anderson et al. 1991, McKee 1992, Duval et al. 1996, Scott 1997, Scott \& McKee 1999, Macias et al. 2002, Olby et al. 2003, Kasakos et al. 2005, Laitinen \& Puerto 2005). Porém, a literatura é escassa quanto aos resultados encontrados em cães com DDIV e sem percepção à dor profunda superior a 48 horas e que não foram submetidos ao tratamento cirúrgico (Davis \& Sharp 1983). Diante disto, o objetivo deste trabalho foi avaliar a recuperação funcional (RF) de cães com diagnóstico de DDIV toracolombar sem percepção à dor profunda, com duração superior a 48 horas e não submetidos ao tratamento cirúrgico atendidos no Hospital Veterinário Universitário (HVU), da Universidade Federal de Santa Maria (UFSM) nos anos de 2002-2010.

\section{MATERIAL E MÉTODOS}

Foram revisados os registros neurológicos de cães com DDIV sem percepção à dor profunda, atendidos entre agosto de 2002 e fevereiro de 2010 no HVU-UFSM. Foram incluídos, neste es- tudo, somente cães com DDIV entre T3-L3 e L4-S3 e que apresentavam paraplegia sem percepção à dor profunda superior a 48 horas. A perda da percepção da dor profunda foi determinada a partir do momento em que os pacientes cessaram os movimentos voluntários (paraplegia) até o atendimento neurológico realizado por um médico veterinário do Serviço de Neurologia do HVU. Outros critérios de inclusão foram: exame clínico e neurológico, diagnóstico de DDIV toracolombar e contato telefônico com os proprietários.

Com base nesses critérios, foram selecionados 37 cães $(n=37)$, alguns sem raça definida $(18,9 \%$ [7/37]) e outros pertencentes às seguintes raças: Teckel (65\% [24/37]), Poodle (8,1\% [3/37]), Pinscher (2,7\% [1/37]), Yorkshire Terrier $(2,7 \%$ [1/37]) e Cocker Spaniel (2,7\% [1/37]). A idade média desses cães foi de 5 anos e a maior parte deles tinha entre três e seis anos de idade. Quanto ao sexo, 51\% (19/37) eram fêmeas e $49 \%$ (18/37) eram machos. A localização da lesão na medula espinhal ocorreu com maior frequência entre T3-L3 (34/37 [92\%]) do que entre L4-S3 (3/37 [8\%]).

O exame neurológico foi constituído de observações gerais (estado mental), avaliação da locomoção, reações posturais, nervos cranianos, reflexos segmentares espinhais, palpação epaxial (hiperestesia) e presença da dor profunda, que foi mensurada pela compressão dos dígitos (periósteo) e da cauda com o auxílio de uma pinça hemostática. A dor profunda foi considerada ausente quando os animais não reagiram ao estímulo doloroso com vocalização, olhar para o local ou inquietude (Sharp \& Wheeler 2005, Dewey 2006). As deficiências neurológicas foram classificadas de acordo com Sharp \& Wheeler (2005): Grau I - somente dor à palpação epaxial; Grau II - ataxia proprioceptiva; Grau III - paraparesia; Grau IV paraplegia com presença de dor profunda; e Grau V paraplegia com ausência de dor profunda. Em relação ao Grau V, a conduta no HVU é a não realização de cirurgia descompressiva da medula espinhal quando a perda de percepção à dor profunda é superior a 48 horas, pois conforme observações de Sharp \& Wheeler (2005), o prognóstico nesses casos é desfavorável.

O diagnóstico de DDIV toracolombar nos pacientes submetidos ao tratamento clínico foi baseado no histórico, raça, idade, sinais clínicos, exames neurológico e radiográfico simples (Dewey 2006). A duração dos sinais clínicos foi definida quando os cães se tornaram paraplégicos sem percepção à dor profunda até a recuperação funcional e classificada em $<30$ dias, entre 30 e 60 dias e >60 dias.

Os proprietários dos cães foram contatados através de telefonemas para a obtenção das seguintes informações: recuperação funcional, recuperação da percepção da dor profunda nos membros afetados, movimento voluntário da cauda, recidiva, presença de disfunção urinária e/ou fecal. Os cães que obtiveram a recuperação funcional retornaram ao HVUUFSM para a realização de um novo exame neurológico. A recuperação funcional foi classificada como satisfatória para cães que recuperaram a habilidade de caminhar sem quedas e insatisfatória para aqueles que não recuperaram a habilidade de caminhar. Os cães que morreram espontaneamente e os que foram submetidos à eutanásia não foram incluídos na avaliação da recuperação funcional.

A relação entre a perda da percepção de dor profunda e a recuperação funcional dos cães foi submetida à análise estatística através do teste qui-quadrado, com nível de significância igual a $5 \%$. 


\section{RESULTADOS E DISCUSSÃO}

Foram localizadas e revisadas 72 fichas clínicas de cães com DDIV e com perda da percepção à dor profunda atendidos entre agosto de 2002 e fevereiro de 2010 no HVUUFSM. Destes, 37 estavam de acordo com os critérios de inclusão e 35 foram excluídos devido à falta de informações nos registros médicos do HVU-UFSM e/ou impossibilidade de estabelecer novo contato por telefone com os proprietários. Desses 37 cães, 17 morreram ou foram submetidos à eutanásia e 20 permaneciam vivos quando do contato telefônico. As informações sobre raça, idade, sexo, localização da lesão na medula espinhal, perda da percepção da dor profunda, duração dos sinais clínicos, recuperação funcional, retorno da percepção da dor profunda, recidivas e eutanásia ou morte dos cães inclusos estão relacionados no Quadro1.

Neste estudo, foi observado que 55\% [11/20] dos cães não submetidos ao tratamento cirúrgico recuperaram-se satisfatoriamente. Entretanto, Davis \& Sharp (1983) verifi- caram que somente $7 \%$ dos cães com DDIV e sem percepção da dor profunda obtiveram melhora funcional quando não submetidos ao tratamento cirúrgico. Resultados semeIhantes aos nossos foram encontrados por Olby et al. (2003) (69\% de recuperação funcional) e Arias et al. (2007) (62\% de recuperação funcional) em cães submetidos ao tratamento cirúrgico para DDIV após 48 horas do início dos sinais clínicos. Diante disso, pode-se dizer que cães com DDIV não submetidos ao tratamento cirúrgico após 48 horas, com perda da percepção à dor profunda, podem apresentar prognóstico semelhante em relação à recuperação funcional dos cães submetidos ao tratamento cirúrgico. Embora os autores deste trabalho sejam favoráveis ao tratamento cirúrgico para cães com DDIV até 48 horas da perda da dor profunda, a resposta satisfatória dos pacientes sem tratamento cirúrgico desta pesquisa ainda deixa dúvidas sobre o benefício da cirurgia após este período.

A duração dos sinais clínicos dos cães que tiveram recuperação satisfatória foi em média de 104 dias, sendo

Quadro 1. Relação da raça, idade, sexo (macho [M], fêmea [F]), localização da lesão (LL), tempo sem percepção da dor profunda (PDP), duração dos sinais clínicos (DSC), recuperação funcional, retorno da percepção da dor profunda (RPDP), recidiva e eutanásia ou morte espontânea de cães com doença do disco intervertebral não submetidos ao tratamento cirúrgico

\begin{tabular}{|c|c|c|c|c|c|c|c|c|c|}
\hline Raça & $\begin{array}{l}\text { Idade } \\
\text { (anos) }\end{array}$ & Sexo & LL & $\begin{array}{l}\text { PDP } \\
\text { (dias) }\end{array}$ & $\begin{array}{c}\text { DSC } \\
\text { (dias) }\end{array}$ & $\begin{array}{l}\text { Recuperação } \\
\text { funcional }\end{array}$ & RPDP & Recidiva & $\begin{array}{c}\text { Eutanásia ou } \\
\text { morte espontânea }\end{array}$ \\
\hline Pinscher & 3 & $M$ & T3-L3 & 30 & 60 & Satisfatória & Presente & Não & - \\
\hline Srd & 5 & M & T3-L3 & 5 & 35 & Satisfatória & Ausente & Não & - \\
\hline Srd & 5 & $\mathrm{~F}$ & T3-L3 & 10 & 40 & Satisfatória & Presente & Não & - \\
\hline Srd & 4 & $\mathrm{~F}$ & T3-L3 & 47 & 62 & Satisfatória & Ausente & Não & - \\
\hline Teckel & 6 & $\mathrm{~F}$ & T3-L3 & 60 & 120 & Satisfatória & Presente & Não & - \\
\hline Teckel & 5 & $M$ & T3-L3 & 5 & 635 & Satisfatória & Presente & Não & - \\
\hline Teckel & 7 & $\mathrm{~F}$ & T3-L3 & 21 & 51 & Satisfatória & Presente & Não & - \\
\hline Teckel & 5 & $\mathrm{~F}$ & T3-L3 & 5 & 29 & Satisfatória & Presente & Não & - \\
\hline Teckel & 5 & $M$ & T3-L3 & 9 & 39 & Satisfatória & Presente & Sim & - \\
\hline Teckel & 7 & $M$ & T3-L3 & 2 & 12 & Satisfatória & Presente & Não & - \\
\hline Teckel & 8 & $\mathrm{~F}$ & T3-L3 & 17 & 47 & Satisfatória & Presente & Não & - \\
\hline Srd & 3 & M & T3-L3 & 70 & - & Insatisfatória & Ausente & - & - \\
\hline Teckel & 4 & $\mathrm{M}$ & T3-L3 & 3 & - & Insatisfatória & Ausente & - & - \\
\hline Teckel & 9 & $M$ & T3-L3 & 5 & - & Insatisfatória & Ausente & - & - \\
\hline Teckel & 8 & M & T3-L3 & 14 & - & Insatisfatória & Ausente & - & - \\
\hline Teckel & 8 & $\mathrm{~F}$ & L4-S3 & 720 & - & Insatisfatória & Ausente & - & - \\
\hline Teckel & 6 & M & T3-L3 & 147 & - & Insatisfatória & Ausente & - & - \\
\hline Teckel & 6 & $\mathrm{~F}$ & T3-L3 & 360 & - & Insatisfatória & Ausente & - & - \\
\hline Teckel & 9 & $\mathrm{~F}$ & T3-L3 & 30 & - & Insatisfatória & Ausente & - & - \\
\hline Yorkshire & 11 & $M$ & T3-L3 & 48 & - & Insatisfatória & Ausente & - & - \\
\hline Cocker & 5 & M & T3-L3 & 7 & 7 & - & - & - & Morte \\
\hline Poodle & 4 & $\mathrm{~F}$ & L4-S3 & 6 & 13 & - & - & - & Eutanásia \\
\hline Poodle & 4 & $\mathrm{~F}$ & T3-L3 & 5 & 35 & - & - & - & Morte \\
\hline Poodle & 5 & $M$ & T3-L3 & 30 & 60 & - & - & - & Eutanásia \\
\hline Srd & 7 & M & T3-L3 & 2 & 4 & - & - & - & Eutanásia \\
\hline Srd & 3 & M & T3-L3 & 3 & 6 & - & - & - & Eutanásia \\
\hline Srd & 9 & $\mathrm{~F}$ & T3-L3 & 20 & 50 & - & - & - & Morte \\
\hline Teckel & 4 & $\mathrm{~F}$ & T3-L3 & 16 & 736 & - & - & - & Eutanásia \\
\hline Teckel & 7 & $M$ & T3-L3 & 15 & 15 & - & - & - & Eutanásia \\
\hline Teckel & 5 & $\mathrm{~F}$ & T3-L3 & 10 & 370 & - & - & - & Eutanásia \\
\hline Teckel & 8 & $M$ & T3-L3 & 2 & 9 & - & - & - & Eutanásia \\
\hline Teckel & 7 & M & T3-L3 & 3 & 10 & - & - & - & Eutanásia \\
\hline Teckel & 4 & $\mathrm{~F}$ & T3-L3 & 7 & 17 & - & - & - & Morte \\
\hline Teckel & 4 & $\mathrm{~F}$ & T3-L3 & 240 & 240 & - & - & - & Eutanásia \\
\hline Teckel & 7 & $\mathrm{~F}$ & T3-L3 & 5 & 35 & - & - & - & Eutanásia \\
\hline Teckel & 4 & $\mathrm{~F}$ & T3-L3 & 10 & 10 & - & - & - & Eutanásia \\
\hline Teckel & 8 & $\mathrm{~F}$ & L4-S3 & 4 & 8 & - & - & - & Eutanásia \\
\hline
\end{tabular}


que $55 \%(6 / 11)$ recuperaram entre 30 e 60 dias. Scott \& Mckee (1999) verificaram a recuperação em um tempo semelhante para cães com DDIV que perderam a dor profunda de 24-48h e que foram tratados cirurgicamente. Um dos cães apresentou duração de sinais clínicos de 635 dias, tempo superior ao estimado por Olby et al. (2004) que considerou o retorno da função motora para alguns pacientes em 270 e 540 dias após o diagnóstico de DDIV. Com isso, os resultados desta pesquisa sugerem aguardar, no mínimo 30 dias para se emitir prognóstico quanto à recuperação funcional de cães com perda da dor profunda acima de 48 horas e não submetidos ao tratamento cirúrgico.

Não houve diferença significativa entre o tempo de perda da dor profunda e a recuperação funcional dos cães com DDIV não submetidos ao tratamento cirúrgico. Outros autores, como Scott 1997, Scott \& McKee 1999 e Kazakos et al. 2005, tentaram relacionar estes dados, mas também não encontraram diferença significativa. A variedade de dados e o pequeno número de amostras dificultaram esta análise.

Foi relatado por Olby et al. (2003) que pacientes com DDIV e perda de dor profunda possuem diferentes graus de comprometimento da medula espinhal e isso pode influenciar na recuperação ou não da habilidade de caminhar. O sucesso do prognóstico quanto à recuperação funcional baseado apenas neste sinal clínico é um método subjetivo, em decorrência disso, torna-se importante associar outros métodos, como a concentração de glutamato no líquido cefalorraquidiano (Olby et al. 1999) e observação de áreas de hiperintensidade da medula espinhal avaliada por ressonância magnética (Ito et al. 2005).

O retorno da percepção à dor profunda ocorreu em $82 \%$ (9/11) dos cães com recuperação funcional satisfatória. Todos os cães que recuperaram a percepção da dor profunda apresentaram o abano voluntário da cauda em resposta ao estímulo dos proprietários. Esta resposta, segundo Olby et al. (2003), foi o primeiro sinal indicativo de recuperação dos cães com lesões da medula espinhal que tiveram retorno da função motora.

Para Olby et al. (2003), a recuperação da função motora em cães com ausência de dor profunda pode ocorrer por desenvolvimento do andar reflexo espinhal. Esta alteração pode ocorrer vários meses após a lesão medular, caracterizado por acentuada incoordenação da marcha e incontinência urinária. Neste estudo, dois cães (18\% [2/11]) retornaram a deambular sem percepção da dor profunda e com sinais clínicos sugestivos de andar espinhal, pois apresentavam ataxia nos membros pélvicos, abano involuntário da cauda e incontinência urinária. Handa et al. (1986) mencionaram que o andar espinhal tem origem num circuito espinhal local e pode ocorrer após a transecção da medula espinhal.

Os distúrbios da micção ocorreram em $40 \%$ dos cães (8/20), quatro (20\% [4/20]) com recuperação funcional insatisfatória desenvolveram incontinência urinária e dois (10\% [2/20]) com recuperação funcional satisfatória, sen- do um deles associado à incontinência fecal. Dois cães (10\% [2/20]) desenvolveram retenção urinária. De acordo com os proprietários, a bexiga dos cães com retenção de urina era drenada por compressão manual três vezes ao dia. De acordo com Olby et al. (2003), 37\% dos cães paraplégicos sem percepção da dor profunda, que tiveram retorno da função motora, permaneceram com incontinência fecal ou urinária.

Neste estudo, dois animais morreram após desenvolver insuficiência renal aguda. Em cães paraplégicos com retenção urinária, a insuficiência renal pode ocorrer secundária às infecções do trato urinário (pielonefrite), como consequência da ausência ou falha no emprego da técnica de compressão da bexiga e sondagem vesical sem assepsia (Dewey 2006). Em outros dois cães as causas da morte foram comprometimento cardiorrespiratório e gastrenterite hemorrágica devido ao uso prolongado de corticosteróides. Para Sharp \& Wheeler (2005) \& Lexmaulová et al. (2008), a gastroenterite hemorrágica é a causa mais frequente de morte em pacientes com DDIV toracolombar.

Apenas um dos pacientes desta pesquisa apresentou recidiva em Grau II um ano depois da sua melhora, restabelecendo sua condição neurológica após 30 dias também sem o tratamento cirúrgico. No estudo realizado por Davis \& Sharp (1983), as recidivas ocorreram em 34\% dos cães não submetidos ao tratamento cirúrgico. Mann et al. (2006) observaram $50 \%$ de recidiva nos pacientes tratados clinicamente entre seis meses e um ano após o tratamento inicial.

Em $61,5 \%$ (8/13) dos cães submetidos à eutanásia, os procedimentos ocorreram em um tempo inferior a 30 dias do início dos sinais clínicos e por determinação dos proprietários. Por outro lado, $55 \%$ das recuperações funcionais satisfatórias ocorreram entre 30 e 60 dias. Portanto, estes resultados demonstram que outros cães poderiam ter recuperado a sua função neurológica caso fosse esperado no mínimo um mês do início dos sinais clínicos (Fig.1).

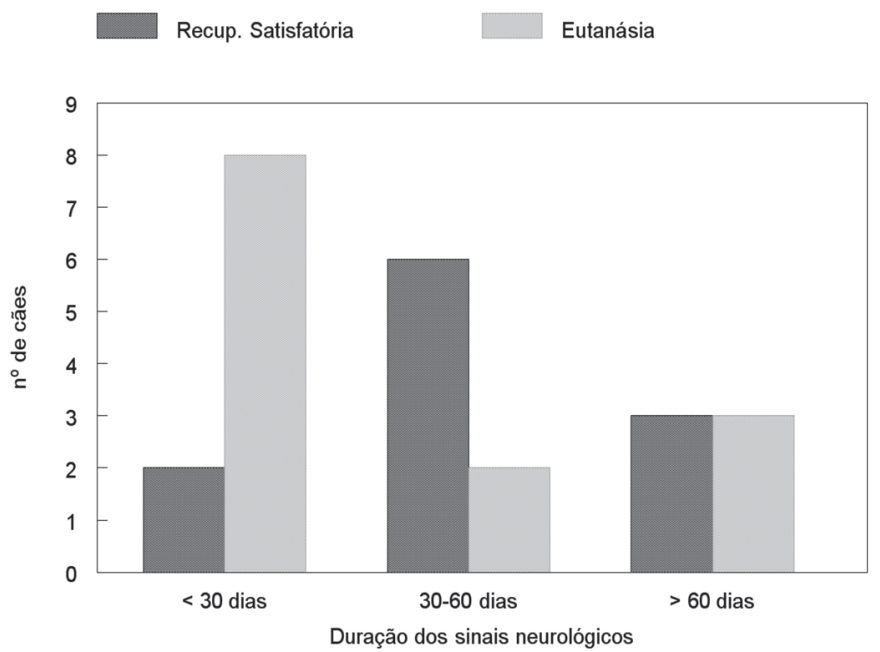

Fig.l. Esquema representativo do número de cães com diagnóstico de DDIV sem percepção à dor profunda superior a 48 horas com recuperação funcional. 


\section{CONCLUSÃO}

De acordo com os resultados obtidos neste estudo, podese concluir que pelo menos a metade dos cães com diagnóstico DDIV sem percepção à dor profunda superior a 48 horas e não submetidos ao tratamento cirúrgico podem apresentar recuperação funcional satisfatória e que é necessário esperar, no mínimo, 30 dias do início dos sinais clínicos para estabelecer um prognóstico quanto ao retorno dos movimentos voluntários.

\section{REFERENCIAS}

Anderson S.M., Lippincott C.L. \& Gill P.J. 1991. Hemilaminectomy in dogs without deep pain perception. Calif. Vet. 45:24-28.

Arias M.V.B., Nishioka C.M., Garcia C.O., Reia A.Z., Júnior D.B. \& Marcasso R.A. 2007. Avaliação dos resultados clínicos após cirurgia descompressiva em cães com doença do disco intervertebral. Arq. Bras. Med. Vet. 59:1445-1450.

Davis J.V. \& Sharp N.J.H. 1983. A comparison of conservative treatment and fenestration for thoracolumbar intervertebral disc disease in the dog. J. Small Anim. Pract. 24:721-729.

Dewey C.W. 2006. Neurologia de Cães e Gatos. Roca, São Paulo. 368p.

Handa Y., Naito A., Watanabe S., Komatsu S. \& Shimizu Y. 1986. Functional recovery of locomotive beravior in the adult spinal dog. Tohoku J. Exp. Med. 148:373-384.

Ito D., Matsunaga S., Jeffery N.D., Sasaky N., Nishimura R., Mochizuki M., Kasahara M., Fujiwara R. \& Ogawa H. 2005. Prognostic value of magnetic resonance imaging in dogs with paraplegia caused by thoracolumbar intervertebral disk extrusion: 77 cases (2000-2003). J. Am. Vet. Med. Assoc. 227:1454-1460.

Kazakos G., Polizopoulou Z.S., Patzicas M.N., Tsimopoulos G., Roubies N. \& Dessiris A. 2005. Duration and severity of clinical sings as prognostic indicators in 30 dogs with thoracolumbar disk disease after surgical decompression. J. Vet. Med. A, Physiol. Pathol. Clin. Med., 52:147-152.

Lecouteur R. \& Grandy J.L. 2004. Doenças da medula espinhal, p.644694. In: Ettinger S.J. \& Feldman E.C. (Eds), Tratado de Medicina Interna de Pequenos Animais. $5^{\underline{a}}$ ed. Guanabara Koogan, Rio de Janeiro.

Laitinen O.M. \& Puerto D.A. 2005. Surgical decompression in dogs with thoracolumbar intervertebral disc disease and loss of deep pain perception: A retrospective study of 46 cases. Acta Vet. Scand. 46:79-85.
Lexmaulová L., Novotná R., Rauser P. \& Necas A. 2008. Complications related to surgical treatment of intervertebral disc in dogs. Acta Vet. Brno, 77:269-276.

Macias C., McKee W.M., May C. \& Innes J.F. 2002. Thoracolumbar disc disease in large dogs: A study of 99 cases. J. Small Anim. Pract. 43:439-446.

Mann F.A., Wagner-Mann C.C., Dunphy E.D., Ruben D.S., Rochat M.C. \& Bartels K.E. 2006. Recurrence rate of presumed thoracolumbar intervertebral disc disease in ambulatory dogs with spinal hiperpathia treated with anti-inflammatory drugs: 78 cases (1997-2000). J. Vet. Emerg. Crit. Care 17:53-60.

McKee W.M. 1992. A comparison of hemilaminectomy (with concomitant disc fenestration) and dorsal laminectomy for the treatment of thoracolumbar disc protrusion in dogs. Vet. Rec. 130:296-300.

Olby J.N., Sharp N.J.H., Muñana K.R. \& Papich M.G. 1999. Chronic and acute compressive spinal cord lesions in dogs due to intervertebral disc herniation are associated with elevation in lumbar cerebrospinal fluid glutamate concentration. J. Neurotrauma 16:12151224.

Olby N., Levine J., Harris T., Munana K., Skeen T. \& Sharp N. 2003. Long-term functional outcome of dogs with severe injuries of the thoracolumbar spinal cord: 87 cases (1996-2001). J. Am. Vet. Med. Assoc. 222:762-769.

Olby N., Harris T., Burr J., Muñana K., Sharp N. \& Keene B. 2004. Recovery of pelvic limb function in dogs following acute intervertebral disc herniations. J. Neurotrauma 21:49-59.

Scott H.W. 1997. Hemilaminectomy for the treatment of thoracolumbar disc disease in the dog: A follow-up study of 40 cases. J. Small Anim. Pract. 38:488-94.

Scott H.W. \& Mckee W.M. 1999. Laminectomy for 34 dogs with thoracolumbar intervertebral disc disease and loss of deep pain perception. J. Small Anim. Pract. 40:417-22.

Seim H.S. 2005. Cirurgia da espinha toracolombar, p.1259-1287. In: Fossum T.W. (Ed.), Cirurgia de Pequenos Animais. $2^{2}$ ed. Roca, São Paulo.

Sharp N.J.H. \& Wheeler S.J. 2005. Small animal spinal disorders: Diagnosis and surgery. $2^{\text {nd }}$ ed. Elsevier Mosby, Philadelphia. 379p.

Taylor S.M. 2006. Distúrbios da locomoção, p.913-1032. In: Couto N. (Ed.), Medicina Interna de Pequenos Animais. $3^{\underline{a}}$ ed. Elsevier, Rio de Janeiro.

Toombs J.P. \& Bauer M.S. 1998. Afecção do disco intervertebral, p.1287-1305. In: Slatter D. (Ed.), Manual de Cirurgia de Pequenos Animais. $2^{\mathrm{a}}$ ed. Manole, São Paulo.

Wheeler S.J. \& Sharp N.J.H. 1999. Diagnóstico e tratamento cirúrgico das afecções espinais do cão e do gato. Manole, São Paulo. 224p. 